\title{
Cost Efficient Narrow Linewidth Laser Transmitter for Coherent Detection
}

\author{
A. Kaszubowska-Anandarajah ${ }^{1}$, A. Mishra ${ }^{2}$, A. Ellis ${ }^{2}$, L. Barry ${ }^{1}$, P. Anandarajah ${ }^{1}$, P. Perry ${ }^{1}$, D. Reid ${ }^{1}$, \\ J. O'Gorman ${ }^{3}$, R. Phelan ${ }^{3}$ and B. Kelly ${ }^{3}$ \\ 1. Research Institute for Networks and Communication Engineering, Dublin City University, Dublin, Ireland \\ e-mail: Aleksandra.kaszubowska@dcu.ie \\ 2. Photonic Systems Group, Tyndall National Institute, University College Cork, Cork, Ireland \\ 3. Eblana Photonics Ltd, Unit 32, Trinity Enterprise Centre, Dublin 2, Ireland
}

\begin{abstract}
Authors present a cost efficient narrow linewidth laser transmitter for future coherent detection systems. The spectral purity of the laser allows the phase modulation of data signals at bit rates as low as $155 \mathrm{Mb} / \mathrm{s}$. (C)2008 Optical Society of America

OCIS codes: (060.1660) Coherent communications; (060.5060) Phase modulation
\end{abstract}

\section{Introduction}

Ever increasing demand for bandwidth pushes telecommunication operators to consider increasing the line rates from 10 to $40 \mathrm{~Gb} / \mathrm{s}$ and beyond. Unfortunately, transmission lines are not optimized for such high data rates resulting in the $40 \mathrm{~Gb} / \mathrm{s}$ signal suffering from fiber impairments, especially chromatic dispersion and polarization mode dispersion. An attractive approach to achieve increased system capacity would be to employ multi-level modulation formats such as Quadrature Phase Shift Keying (QPSK). These, combined with coherent detection and polarization multiplexing, can increase the data rate without increasing the symbol rate. However, this improvement comes at a price of increased system complexity and cost. In order to efficiently demodulate signals, a coherent receiver either needs to use a local oscillator (LO) locked to the carrier phase or recover the carrier phase after intradyne detection [1]. The first method of demodulation is traditionally difficult to fulfill, as the optical phase locked loop requires ultra-narrow linewidth lasers, which are widely believed to be too costly [2]. Standard lasers such as distributed feedback (DFB) and distributed Bragg reflector (DBR) would not be suitable since coherent systems require linewidths better than 0.001 times the bit rate to achieve optimum performance [2]. This implies that the linewidth requirements are more demanding for lower bit rate systems.

In this paper we demonstrate an inexpensive laser transmitter with a very narrow linewidth that enables coherent detection of data signals using a simple phase lock loop and a single receiver at bit rates $155 \mathrm{Mb} / \mathrm{s}$ and higher. We compare this laser to commercially available DFB and fiber lasers.

\section{Experimental set-up and results}

The laser transmitter focused on was a $1.5 \mu \mathrm{m}$ Discrete Mode (DM) laser, while a commercially available DFB and fiber laser were used for benchmarking. A DM laser is a device, based on a Fabry-Perot structure, which achieves single mode operation by introducing perturbations in the refractive index of the cavity. These perturbations alter the threshold gain values of the individual cavity modes. When the device is biased above threshold, emission occurs at the mode that has the lowest threshold gain. DM laser diodes are capable of single mode operation over wide temperature ranges and exhibit Side Mode Suppression Ratios (SMSR) in excess of $40 \mathrm{~dB}$ [3]. These lasers not only exhibit narrow linewidths but also involve structures that are cheaper and easier to manufacture and differ significantly from that of a DFB laser [4].

Initially, the linewidths of the three lasers (DM, DFB and fiber laser) were measured using the standard delayed self-heterodyne technique with $50 \mathrm{~km}$ of fiber in one arm of the interferometer to achieve resolution better than 3 $\mathrm{kHz}$ [5]. The light in the other arm was phase modulated with a $1 \mathrm{GHz}$ sine wave. The interferometer output was photo-detected and displayed using an RF spectrum analyzer. The linewidths of the lasers were measured to be 7 $\mathrm{MHz}$ (DFB) and $7 \mathrm{kHz}$ (fiber laser) and $320 \mathrm{kHz}$ (DM laser) and are shown in Figure 1 (a).

Having measured the linewidth of the lasers, we went on to verify the usefulness of each of the three lasers for transmission of phase modulated signals. The experimental set-up used is shown in Figure 1(b). The output from the laser transmitter was passed through an isolator (to ensure that the optical feedback does not influence the operation of the laser) and split using a 50:50 coupler. The light in the lower branch of the coupler was passed through a 


\section{CThJJ3.pdf}

polarization controller and modulated with a Pseudo Random Bit Sequence (PRBS) of pattern length of $2^{7}-1$ using a phase modulator. The light from the upper branch of the coupler, used as the LO, was transmitted over $12 \mathrm{~km}$ of single mode fiber in order to de-correlate the phase noise in both branches. It was then combined with the data signal using a 50:50 coupler, detected using a photodiode and the resulting eye diagram was displayed on an oscilloscope. In order to avoid signal fading, due to phase walk off between the LO and the data signal, a phase tracking loop was introduced. To this effect, $10 \%$ of the combined light was directed to a low speed photodiode using a 90:10 optical coupler. The output of the detector was analyzed using a computer equipped with a data acquisition card (DAQ) and the phase of the LO was then adjusted using a fiber stretcher consisting of an electrical amplifier and a piezoelectric element.

(a)

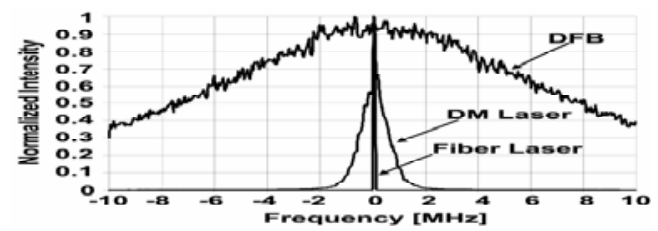

(b)

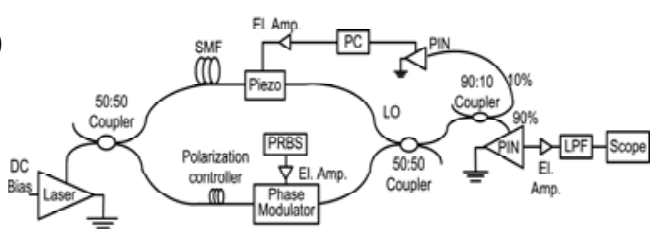

Figure 1: (a) Experimental set-up, (b) linewidth of laser transmitters used

The bit rate was varied between $155 \mathrm{Mb} / \mathrm{s}$ and $3.2 \mathrm{~Gb} / \mathrm{s}$ and some of the received eye diagrams are shown in Figure 2. From the figures it can be seen that the fiber laser performed best, giving a very clear eye for all the bit rates. The signal quality from the DM laser, slightly worse than the fiber laser, still yielded an open eye even at the lowest bit rate. From the diagrams it can also be seen that the relatively large linewidth of the DFB laser makes it unsuitable for transmitting data even at $3.2 \mathrm{~Gb} / \mathrm{s}$ bit rate.
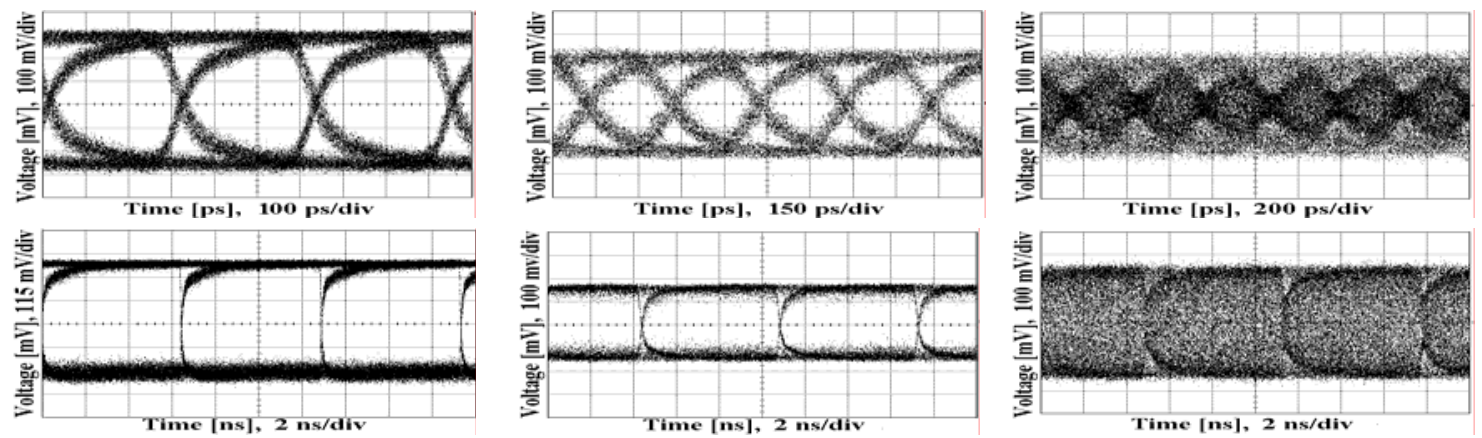

Figure 2: Eye diagrams for the: (left) fiber laser, (centre) DM laser and (right) DFB laser; at (top) $3.2 \mathrm{~Gb} / \mathrm{s}$ and (bottom) $155 \mathrm{Mbit} / \mathrm{s}$

\section{Conclusions}

In this paper, we have shown that the narrow linewidth of low cost Discrete Mode lasers enables true homodyne coherent detection at bit rates of $155 \mathrm{Mbit} / \mathrm{s}$ and above. This simple FP like structure, manufactured using standard optical lithography, possesses very narrow linewidth which enables the transmission of phase modulated data signal. This alleviates the need for digital signal processing based carrier phase recovery in intra-dyne detection systems, greatly simplifying the receiver architecture. The development of such cost efficient coherent detection, based on simple light sources with high spectral purity, could enable the widespread utilization of all the possible benefits of coherent transmission, such as increased spectral efficiency, improved immunity to noise and fiber impairments.

\section{References}

[1] D.S. Ly-Gagnon, S. Tsukamoto, K. Katoh and K. Kikuchi, "Coherent Detection of Optical Quadrature Phase-Shift Keying Signals With Carrier Phase Estimation“, JLT, 24, 12-21, (2006)

[2] T. Pfau, S. Hoffmann, R. Peveling, S. Bhandare, O. Adamczyk, M. Porrmann, R. Noe and Y. Achiam, "1.6 Gbit/s Real-Time Synchronous QPSK Transmission with Standard DFB lasers“ in Proceedings European Conference on Optical Communication, (Sociéte de l'Electricité, de l'Electronique et des Technologies de l'Information et de la Communication, Cannes, 2006) pp.: 65-66

[3] J. Patchell D. Jones, B. Kelly, J. O'Gorman, "Specifying the wavelength and temperature tuning range of a Fabry-Perot laser containing refractive index perturbations," in Proceedings SPIE 5825, 1, (2005)

[4] B. Kelly, R. Phelan, D. Jones, C. Herbert, J. O'Carroll, M. Rensing, J. Wendelboe, C.B. Watts, A. Kaszubowska-Anandarajah, P. Perry, C. Guignard, L.P. Barry and J. O'Gorman, "Discrete mode laser diodes with very narrow linewidth emission", Electronic Letts., 43, (2006)

[5] K. M. Abramski, W. Rodzen, P. R. Kaczmarek, L. Czurak, A. Budnicki, "Laser Diode Linewidth Measurements", in Proceedings Conference on Lasers and Electro-Optics, pp.: 87-90, (2005T) 